\title{
Auditoria Médica y docencia en Medicina Familiar, un estudio antes y después en el Hospital de Pedro Vicente Maldonado
}

\author{
Fausto Gady Torres[1], Carlos Troya[2], Sergio Carrasco[1] \\ 1. Makroscopio Servicios de Salud
}

2. Hospital Hesburgh

DOI: https://doi.org/10.23936/pfr.v2i1.58

PRÁCTICA FAMILIAR RURAL | Vol.2 | No.1 | Marzo 2017 | Recibido: 07/12/2016 | Aprobado: 20/02/2017

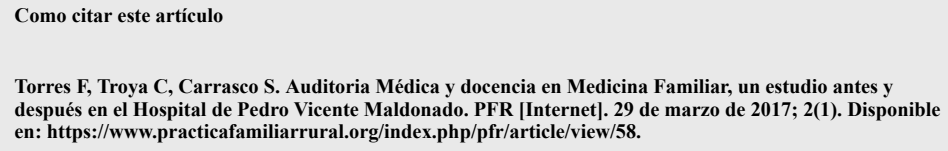

\section{Resumen}

Objetivo: Evaluar la intervención de Auditoria Medica en un grupo residentes de posgrado de Medicina Familiar.

Materiales y métodos: Se realizó un estudio exploratorio, en donde se incluyó una cohorte de médicos y fueron evaluados en un periodo de 6 meses. Se incluyeron 1060 revisiones de actuaciones clínicas y se aplicó el formulario de auditoria medica de terreno validado en Ecuador. La inclusión de casos fue aleatoria inicialmente y posteriormente intencionada, de acuerdo a los procesos de reauditoría, esto para garantizar mayor efectividad.

Resultados y discusión: Se revisaron cuentes principalmente ambulatorias, el nivel de conflictividad fue prácticamente nulo, existió aceptación del proceso de auditoria médica en un ambiente docente, el porcentaje de apelaciones fue bajo (menos del $0,5 \%$ ) y la presentación de casos de interés genero reuniones proactivas, con alta madurez profesional y de discusión científica.

En la evaluación clínica, se inicia con un puntaje promedio de calidad de $70 \%$ y se finaliza con $88 \%$. Se lograron mejoras en casi todos los campos, especialmente en manejo no farmacológico y Captación activa del riesgo de los pacientes. En el manejo legal de la información clínica, se parte de un puntaje alto (más del $90 \%$ ) debido a que los formularios son electrónicos y tienen controles de registro.

Conclusiones: La tendencia observada es de mejoramiento, gracias a la personalización de las intervenciones por la administración del hospital, los residentes y Auditoria.

La aceptación del proceso por parte de los auditados es buena, gracias al enfoque cooperativo y no punitivo en un contexto académico.

Estos resultados son alentadores y confirman la validez, efectividad y eficiencia de los procesos de evaluación y monitoreo de la Calidad a través de Auditoria Medica integral en un medio docente. Es importante resaltar que el proceso debe ser continuo para lograr efectividad de la intervención.

\section{Palabras clave}

Auditoría médica, docencia, mejoramiento continuo, calidad

Medical Audit and Teaching in Family Medicine, a Before and After Study at Hospital Pedro Vicente Maldonado

\section{Abstract}

Objective: To evaluate the intervention of a Medical Audit in a group of postgraduate Family Medicine residents.

Materials and Methods: An exploratory study was carried out, in which a cohort of physicians was evaluated over 6 months. The researchers included 1,060 clinical action reviews and applied the validated onsite medical audit form in Ecuador. The inclusion of cases was initially randomized and subsequently intentional, according to the re-audit processes, to ensure greatest effectiveness. 
Results and Discussion: Ambulatory accounts were reviewed primarily. The level of conflict was practically zero; there was acceptance of the medical audit process in a teaching environment; the percentage of appeals was low (less than $0.5 \%$ ); and cases were presented with high professional maturity and provoked scientific discussion.

The clinical evaluation started with an average quality score of $70 \%$ and ended with $88 \%$. Improvements were achieved in almost all fields, especially in non-pharmacological management and active identification and intervention of patient risk. In the legal management of clinical information, a high score (more than 90\%) is based on the fact that the forms are electronic and have registration controls.

Conclusions: The observed trend is one of improvement, due to the personalization of interventions by hospital administration and residents.

The acceptance of the process by the residents is high, due to the cooperative, non-punitive approach in an academic context.

These results are encouraging and confirm the validity, effectiveness, and efficiency of the quality assessment and monitoring processes through a comprehensive medical audit in a teaching environment. It is important to emphasize that the process must be continuous to achieve effectiveness of the intervention.

\section{Keywords}

Medical audit, teaching, continuous improvement, quality

\section{Introducción}

Los sistemas de Garantía de Calidad en Salud son indispensables, la gestión a nivel de los establecimientos sanitarios es un reto, debe ser contextualizada y con lineamientos enfocados en el mejoramiento continuo de la calidad, para la intervención en el Hospital Pedro Vicente Maldonado, se utiliza una metodología adaptada de la supervisión participativa, modelizada con principios de garantía de calidad, mejora continua, no punitiva, construcción de competencias y excelencia 1-7, este proceso que ha sido validado en Ecuador y en múltiples instituciones públicas y privadas, se caracteriza por la redefinición del concepto clásico de la auditoria médica, con un cambio del imaginario medico de un proceso de control estricto evolucionándolo hacia un proceso cooperativo de mejoramiento de la calidad y en una herramienta para la toma de decisiones del gerente, la Auditoría Médica como se la plantea en esta intervención, tiene injerencia en todos los aspectos de la gestión 1,2,8-10.

El presente trabajo se desarrolló en el Hospital Docente Pedro Vicente Maldonado, ubicado en el cantón homónimo de Pichincha, el mismo que brinda servicios de salud a los habitantes de las zonas rurales del Nor-Occidente de Pichincha. Dicho hospital tiene como principales usuarios de sus servicios a los beneficiarios del Instituto Ecuatoriano de Seguridad Social y del Seguro Social Campesino. Desde el año 2009 el hospital cuenta con un convenio con la Pontificia Universidad Católica de Quito a través del cual se participa en la formación de Médicos de Familia, con la finalidad de promover la anidación de los profesionales en las zonas rurales y mantener elevados niveles de calidad de atención de salud. El Hospital cuenta con una estructura docente que permite que los residentes participen en actividades autónomas y también actividades de atención supervisadas en consulta externa y hospitalización, de igual manera, existen ambientes de formación académica. Los residentes tienen rotaciones en hospitales y otras instituciones de salud de la ciudad de Quito en diferentes especialidades de acuerdo al su programa de postgrado y además cuentan con la rotación en el hospital de Pedro Vicente Maldonado.

Con la finalidad de favorecer la calidad de atención el hospital desarrolló algunas estrategias para brindar soporte a los residentes, una de ellas es atención médica supervisada por un médico tratante responsable de la consulta externa y hospitalización, así como la implementación de guías de práctica clínica desarrolladas por médicos de familia y socializadas entre los residentes a través de documentos físicos. Posteriormente se decidió involucrar el proceso de auditoria médica entre las estrategias docentes de la institución.

\section{Objetivos}

Evaluar la intervención de Auditoria Medica en un grupo residentes de posgrado de Medicina Familiar.

\section{Materiales y Métodos}

Se realizó un estudio de intervención "Antes y después", basados en criterios de calidad clínica y con procesos de monitoreo permanentes. Se incluyeron 1060 casos en un periodo de 6 meses, se tomaron los 2 primeros meses como una fase de diagnóstico $(\mathrm{n}=346)$ y los 4 meses subsiguientes como fase de intervención / resultados $(\mathrm{n}=714)$.

Instrumentalmente, se utilizó la guía de campo de auditoria de terreno, la misma que es eficiente ya que actúa como informe individual de auditoria, se registran en ella todos los problemas encontrados y las recomendaciones, así como las posibles apelaciones, además es un documento que evidencia la auditoria y es firmado por el auditor y un representante de los auditados. El instrumento fue desarrollado por Torres a partir de una base teórica política en Ecuador, se ha validado en terreno y ha demostrado ser eficiente y efectivo para evaluar la historia clínica. 11,12 Esta guía de campo tiene 2 partes que dan un puntaje, la pertinencia clínica y el riesgo legal. Cada una de ellas consta de varios ítems, los cuales están ponderados por la importancia que tengan. Al final se otorgará un peso a la parte clínica y otro al riesgo legal que en conjunto será el puntaje global de la auditoria de terreno realizada. La importancia de la ponderación radica en que el auditado tiene un documento objetivo de su evaluación y conocerá en que específicamente fue el error. Por lo tanto, tiene un referente para el proceso continuo de control. 


\section{Descriptores de los casos}

Se analizaron 1060 casos de atenciones sanitarias de residentes de Medicina Familiar del Hospital Pedro Vicente Maldonado, la mayoría correspondieron a atenciones de emergencia (60\%), seguidas por atenciones hospitalarias clínicas y consulta externa $(20$ y $18 \%)$, las hospitalizaciones quirúrgicas representaron un $2 \%$.

\section{Intervención de auditoria médica en docencia de Medicina Familiar}

El proceso de auditoria medica se planteó como una estrategia de gestión de la calidad, al intervenir un grupo de estudiantes de Medicina Familiar, el nivel de conflictividad fue prácticamente nulo, quizás debido a que el componente de docencia de la estrategia de supervisión participativa se pudo desarrollar a su máxima capacidad.

Las ventajas de incluir gestión de calidad como una estrategia docente, radican en que el estudiante mira el aprendizaje desde casos reales y con aspectos técnicos, administrativos y legales que son parte natural de las evaluaciones de auditoria medica; se visualiza el control de calidad como un proceso más que aporta en el aprendizaje.

\section{Análisis de pertinencia de todo el proceso}

Se realizaron 2 tipos de análisis en esta intervención, el instrumento arrojó cumplimientos de indicadores individualmente, pero también realizó un análisis cuantitativo del puntaje (sobre 100 puntos) de la atención. El puntaje fue ponderado y respondió al instrumento de auditoria médica utilizado. Se analizó el comportamiento de los no cumplimientos por mes y se observó una tendencia hacia la disminución de los mismos conforme avanzó la intervención de auditoria médica, los aspectos de evaluación de la integralidad parten altos y tienen un mejoramiento importante.

Tabla 1: Porcentaje de incumplimientos por mes

\begin{tabular}{|c|c|c|c|c|c|c|c|c|c|c|c|c|c|}
\hline & & \multicolumn{2}{|c|}{ OCT } & \multicolumn{2}{|c|}{ NOV } & \multicolumn{2}{|c|}{ DIC } & \multicolumn{2}{|c|}{ ENE } & \multicolumn{2}{|c|}{ FEB } & \multicolumn{2}{|c|}{ MAR } \\
\hline $\begin{array}{l}\text { CRITERIO CLÍNICO DE } \\
\text { LA REFERENCIA }\end{array}$ & $\begin{array}{l}\text { NO CUMPLE } \\
\text { CUMPLE }\end{array}$ & $\begin{array}{c}7 \\
194\end{array}$ & $3 \%$ & $\begin{array}{c}5 \\
140\end{array}$ & $3 \%$ & $\begin{array}{r}10 \\
157\end{array}$ & $6 \%$ & $\begin{array}{c}6 \\
204\end{array}$ & $3 \%$ & $\begin{array}{c}7 \\
145\end{array}$ & $5 \%$ & $\begin{array}{c}4 \\
181\end{array}$ & $2 \%$ \\
\hline $\begin{array}{l}\text { DOCUMENTACIÓN DE } \\
\text { LA REFERENCIA }\end{array}$ & $\begin{array}{l}\text { NO CUMPLE } \\
\text { CUMPLE }\end{array}$ & $\begin{array}{c}11 \\
190\end{array}$ & $5 \%$ & $\begin{array}{c}14 \\
131\end{array}$ & $10 \%$ & $\begin{array}{r}15 \\
152\end{array}$ & $9 \%$ & $\begin{array}{c}14 \\
196\end{array}$ & $7 \%$ & $\begin{array}{c}0 \\
152\end{array}$ & $0 \%$ & $\begin{array}{c}0 \\
185\end{array}$ & $0 \%$ \\
\hline $\begin{array}{l}\text { DIAGNOSTICO } \\
\text { PERTINENTE }\end{array}$ & $\begin{array}{l}\text { NO CUMPLE } \\
\text { CUMPLE }\end{array}$ & $\begin{array}{c}48 \\
153\end{array}$ & $24 \%$ & $\begin{array}{c}18 \\
127\end{array}$ & $12 \%$ & $\begin{array}{c}50 \\
117\end{array}$ & $30 \%$ & $\begin{array}{c}46 \\
164\end{array}$ & $22 \%$ & $\begin{array}{c}22 \\
130\end{array}$ & $14 \%$ & $\begin{array}{c}39 \\
146\end{array}$ & $21 \%$ \\
\hline $\begin{array}{l}\text { TRATAMIENTO } \\
\text { PERTINENTE }\end{array}$ & $\begin{array}{l}\text { NO CUMPLE } \\
\text { CUMPLE }\end{array}$ & $\begin{array}{c}55 \\
146\end{array}$ & $27 \%$ & $\begin{array}{c}28 \\
117\end{array}$ & $19 \%$ & $\begin{array}{c}53 \\
114\end{array}$ & $32 \%$ & $\begin{array}{c}47 \\
163\end{array}$ & $22 \%$ & $\begin{array}{c}28 \\
124\end{array}$ & $18 \%$ & $\begin{array}{c}26 \\
159\end{array}$ & $14 \%$ \\
\hline $\begin{array}{l}\text { MEDICINAS } \\
\text { PERTINENTES }\end{array}$ & $\begin{array}{l}\text { NO CUMPLE } \\
\text { CUMPLE }\end{array}$ & $\begin{array}{c}54 \\
147\end{array}$ & $27 \%$ & $\begin{array}{c}8 \\
137\end{array}$ & $6 \%$ & $\begin{array}{c}50 \\
117\end{array}$ & $30 \%$ & $\begin{array}{c}43 \\
167\end{array}$ & $20 \%$ & $\begin{array}{c}18 \\
134\end{array}$ & $12 \%$ & $\begin{array}{c}22 \\
163\end{array}$ & $12 \%$ \\
\hline IMAGEN PERTINENTE & $\begin{array}{l}\text { NO CUMPLE } \\
\text { CUMPLE }\end{array}$ & $\begin{array}{c}20 \\
181\end{array}$ & $10 \%$ & $\begin{array}{c}12 \\
133\end{array}$ & $8 \%$ & $\begin{array}{c}31 \\
136\end{array}$ & $19 \%$ & $\begin{array}{c}17 \\
193\end{array}$ & $8 \%$ & $\begin{array}{c}6 \\
146\end{array}$ & $4 \%$ & $\begin{array}{c}10 \\
175\end{array}$ & $5 \%$ \\
\hline $\begin{array}{l}\text { LABORATORIO } \\
\text { PERTINENTE }\end{array}$ & $\begin{array}{l}\text { NO CUMPLE } \\
\text { CUMPLE }\end{array}$ & $\begin{array}{c}35 \\
166\end{array}$ & $17 \%$ & $\begin{array}{c}11 \\
134\end{array}$ & $8 \%$ & $\begin{array}{c}34 \\
133\end{array}$ & $20 \%$ & $\begin{array}{c}32 \\
178\end{array}$ & $15 \%$ & $\begin{array}{c}11 \\
141\end{array}$ & $7 \%$ & $\begin{array}{c}6 \\
179\end{array}$ & $3 \%$ \\
\hline $\begin{array}{l}\text { PRESCRIPCIÓN } \\
\text { COMPLETA }\end{array}$ & $\begin{array}{c}\text { CUMPLE } \\
\text { NO CUMPLE }\end{array}$ & $\begin{array}{c}49 \\
152\end{array}$ & $24 \%$ & $\begin{array}{c}1 \\
144\end{array}$ & $1 \%$ & $\begin{array}{c}23 \\
144\end{array}$ & $14 \%$ & $\begin{array}{c}49 \\
161\end{array}$ & $23 \%$ & $\begin{array}{c}49 \\
103\end{array}$ & $32 \%$ & $\begin{array}{c}37 \\
148\end{array}$ & $20 \%$ \\
\hline $\begin{array}{l}\text { USO DE GENÉRICOS } \\
\text { EN PRESCRIPCIÓN }\end{array}$ & $\begin{array}{c}\text { CUMPLE } \\
\text { NO CUMPLE }\end{array}$ & $\begin{array}{c}106 \\
95\end{array}$ & $53 \%$ & $\begin{array}{l}97 \\
48\end{array}$ & $67 \%$ & $\begin{array}{c}34 \\
133\end{array}$ & $20 \%$ & $\begin{array}{c}37 \\
173\end{array}$ & $18 \%$ & $\begin{array}{c}0 \\
152\end{array}$ & $0 \%$ & $\begin{array}{c}4 \\
181\end{array}$ & $2 \%$ \\
\hline $\begin{array}{l}\text { MANEJO NO } \\
\text { FARMACOLÓGICO }\end{array}$ & $\begin{array}{c}\text { CUMPLE } \\
\text { NO CUMPLE }\end{array}$ & $\begin{array}{c}97 \\
104\end{array}$ & $48 \%$ & $\begin{array}{c}113 \\
32\end{array}$ & $78 \%$ & $\begin{array}{c}102 \\
65\end{array}$ & $61 \%$ & $\begin{array}{c}67 \\
143\end{array}$ & $32 \%$ & $\begin{array}{c}13 \\
139\end{array}$ & $9 \%$ & $\begin{array}{c}20 \\
165\end{array}$ & $11 \%$ \\
\hline $\begin{array}{l}\text { CAPTACIÓN ACTIVA } \\
\text { DE RIESGOS }\end{array}$ & $\begin{array}{c}\text { CUMPLE } \\
\text { NO CUMPLE }\end{array}$ & $\begin{array}{r}90 \\
111\end{array}$ & $45 \%$ & $\begin{array}{c}109 \\
36\end{array}$ & $75 \%$ & $\begin{array}{l}90 \\
77\end{array}$ & $54 \%$ & $\begin{array}{c}77 \\
133\end{array}$ & $37 \%$ & $\begin{array}{c}19 \\
133\end{array}$ & $13 \%$ & $\begin{array}{c}29 \\
156\end{array}$ & $16 \%$ \\
\hline
\end{tabular}

Se analizó también el comportamiento del puntaje de auditoria médica, encontrando que el promedio de inicio fue menor que el de los meses finales, lo que indica un mejoramiento en el proceso de atención, esa tendencia fue significativa $(\mathrm{p}=0.0000)$

Tabla 2: Análisis de puntajes porcentuales de la calidad de la atención

$\begin{array}{lccccccccc} & \text { Obs } & \text { Medias } & \begin{array}{c}\text { Desviación } \\ \text { estándar }\end{array} & \text { Min } & \mathbf{2 5 \%} & \text { Mediana } & \mathbf{7 5 \%} & \text { Max } & \text { Modo } \\ \text { 1. OCTUBRE } & 201 & 70,9241 & 19,774 & 15 & 58 & 73,25 & 88 & 100 & 88 \\ \text { 2. NOVIEMBRE } & 145 & 67,6793 & 15,0144 & 30,25 & 61 & 64 & 81,75 & 100 & 64 \\ \text { 3. DICIEMBRE } & 167 & 68,7994 & 20,5127 & 19 & 51 & 70 & 88 & 100 & 100 \\ \text { 4. ENERO } & 210 & 77,7536 & 18,4942 & 10 & 66 & 79,25 & 93 & 100 & 100\end{array}$




$\begin{array}{lccccccccc}\text { 5. FEBRERO } & 152 & 88,426 & 16,2772 & 30,75 & 82 & 91,25 & 100 & 100 & 100 \\ \text { 6. MARZO } & 183 & 88,4194 & 14,4339 & 21,25 & 81 & 91,25 & 100 & 100 & 100\end{array}$

\section{Evaluación antes y después: Análisis categórico de los indicadores}

Se analizaron los ítems evaluados en el terreno y se observó que exceptuando uno, todos tuvieron mejoría medidos como la diferencia del porcentaje de incumplimiento de la fase de diagnóstico versus la fase pos intervención, los valores de mejoría muy bajos corresponden a indicadores que fueron óptimos desde el inicio. Vale la pena recalcar que las variables relacionadas con atención integral tuvieron mayor mejoría que las otras, esto quizás debido a la característica de los postgradistas.

Tabla 3: Evaluación de ítems evaluados según pre o post intervención

\begin{tabular}{|c|c|c|c|c|c|c|}
\hline \multirow{3}{*}{$\begin{array}{l}\text { CRITERIO CLÍNICO } \\
\text { DE LA REFERENCIA }\end{array}$} & & \multicolumn{2}{|c|}{ Diagnostico } & \multicolumn{2}{|c|}{ Post intervención } & \multirow{3}{*}{$\begin{array}{c}\text { DIFERENCIA DE } \\
\text { PORCENTAJES } \\
0,2 \%\end{array}$} \\
\hline & $\begin{array}{l}\text { NO } \\
\text { CUMPLE }\end{array}$ & 12 & \multirow{2}{*}{$3 \%$} & & \multirow[t]{2}{*}{$3 \%$} & \\
\hline & CUMPLE & 334 & & 326 & & \\
\hline \multirow{2}{*}{$\begin{array}{l}\text { DOCUMENTACIÓN DE } \\
\text { LA REFERENCIA }\end{array}$} & $\begin{array}{l}\text { NO } \\
\text { CUMPLE }\end{array}$ & 25 & \multirow[t]{2}{*}{$7 \%$} & 0 & \multirow[t]{2}{*}{$0 \%$} & \multirow[t]{2}{*}{$7,2 \%$} \\
\hline & CUMPLE & 321 & & 337 & & \\
\hline \multirow{2}{*}{$\begin{array}{l}\text { DIAGNOSTICO } \\
\text { PERTINENTE }\end{array}$} & $\begin{array}{l}\text { NO } \\
\text { CUMPLE }\end{array}$ & 66 & \multirow[t]{2}{*}{$19 \%$} & 61 & \multirow[t]{2}{*}{$18 \%$} & \multirow[t]{2}{*}{$1,0 \%$} \\
\hline & CUMPLE & 280 & & 276 & & \\
\hline \multirow{2}{*}{$\begin{array}{l}\text { TRATAMIENTO } \\
\text { PERTINENTE }\end{array}$} & $\begin{array}{l}\text { NO } \\
\text { CUMPLE }\end{array}$ & 83 & \multirow[t]{2}{*}{$24 \%$} & 54 & \multirow[t]{2}{*}{$16 \%$} & \multirow[t]{2}{*}{$8,0 \%$} \\
\hline & CUMPLE & 263 & & 283 & & \\
\hline \multirow{2}{*}{$\begin{array}{l}\text { MEDICINAS } \\
\text { PERTINENTES }\end{array}$} & $\begin{array}{l}\text { NO } \\
\text { CUMPLE }\end{array}$ & 62 & \multirow[t]{2}{*}{$18 \%$} & 40 & \multirow[t]{2}{*}{$12 \%$} & \multirow[t]{2}{*}{$6,0 \%$} \\
\hline & CUMPLE & 284 & & 297 & & \\
\hline \multirow[t]{2}{*}{ IMAGEN PERTINENTE } & $\begin{array}{l}\text { NO } \\
\text { CUMPLE }\end{array}$ & 32 & \multirow[t]{2}{*}{$9 \%$} & & \multirow[t]{2}{*}{$5 \%$} & \multirow[t]{2}{*}{$4,5 \%$} \\
\hline & CUMPLE & 314 & & 321 & & \\
\hline \multirow{2}{*}{$\begin{array}{l}\text { LABORATORIO } \\
\text { PERTINENTE }\end{array}$} & $\begin{array}{l}\text { NO } \\
\text { CUMPLE }\end{array}$ & 46 & \multirow[t]{2}{*}{$13 \%$} & 17 & \multirow[t]{2}{*}{$5 \%$} & \multirow[t]{2}{*}{$8,3 \%$} \\
\hline & CUMPLE & 300 & & 320 & & \\
\hline \multirow{2}{*}{$\begin{array}{l}\text { PRESCRIPCIÓN } \\
\text { COMPLETA }\end{array}$} & $\begin{array}{l}\text { NO } \\
\text { CUMPLE }\end{array}$ & 50 & \multirow[t]{2}{*}{$14 \%$} & 86 & $26 \%$ & $-11,1 \%$ \\
\hline & CUMPLE & 296 & & 251 & & \\
\hline USO DE GENÉRICOS & $\begin{array}{l}\text { NO } \\
\text { CUMPLE }\end{array}$ & 203 & $59 \%$ & 4 & $1 \%$ & $57,5 \%$ \\
\hline & CUMPLE & 143 & & 333 & & \\
\hline USO DE ESTÁNDAR & $\begin{array}{l}\text { NO } \\
\text { CUMPLE }\end{array}$ & 4 & $1 \%$ & 1 & $0 \%$ & $0,9 \%$ \\
\hline & CUMPLE & 342 & & 336 & & \\
\hline MANEJO NO & $\begin{array}{l}\text { NO } \\
\text { CUMPLE }\end{array}$ & 210 & $61 \%$ & 33 & $10 \%$ & $50,9 \%$ \\
\hline & CUMPLE & 136 & & 304 & & \\
\hline CAPTACIÓN ACTIVA & $\begin{array}{l}\text { NO } \\
\text { CUMPLE }\end{array}$ & 199 & $58 \%$ & 48 & $14 \%$ & $43,3 \%$ \\
\hline & CUMPLE & 147 & & 289 & & \\
\hline POLIFARMACIA & $\begin{array}{l}\text { NO } \\
\text { CUMPLE }\end{array}$ & 285 & $82 \%$ & 270 & $80 \%$ & $2,3 \%$ \\
\hline & CUMPLE & 61 & & 67 & & \\
\hline
\end{tabular}

Estas diferencias de porcentajes nos permiten visualizar una mejora gracias a la intervención, se realiza una regresión logística para mirar la significancia e influencia de dichos indicadores analizados y encontramos que los relacionados a integralidad tienen la fuerza de asociación más alta (OR) y son inferenciables.

Tabla 4: Análisis inferencial de indicadores 


\begin{tabular}{lccccc}
\hline & & & & Inferior & Superior \\
CRITERIO CLÍNICO DE LA REFERENCIA &, 977 &, 323 &, 424 &, 077 & 2,326 \\
DIAGNOSTICO PERTINENTE &, 022 &, 883 &, 949 &, 470 & 1,915 \\
TRATAMIENTO PERTINENTE & 5,054 &, 025 &, 355 &, 144 &, 876 \\
MEDICINAS PERTINENTES &, 231 &, 631 & 1,245 &, 509 & 3,048 \\
\hline IMAGEN PERTINENTE &, 146 &, 703 & 1,256 &, 389 & 4,058 \\
LABORATORIO PERTINENTE & 8,433 &, 004 & 4,115 & 1,584 & 10,692 \\
PRESCRIPCIÓN COMPLETA & 10,075 &, 002 &, 341 &, 176 &, 663 \\
\hline USO DE GENÉRICOS EN PRESCRIPCIÓN & 73,628 &, 000 & 166,243 & 51,700 & 534,559 \\
\hline MANEJO NO FARMACOLÓGICO & 63,346 &, 000 & 10,303 & 5,801 & 18,298 \\
\hline CAPTACIÓN ACTIVA DE RIESGOS & 33,924 &, 000 & 5,631 & 3,148 & 10,072 \\
\hline POLIFARMACIA & 6,042 &, 014 & 2,606 & 1,214 & 5,592
\end{tabular}

Evaluación antes y después: Análisis del puntaje de auditoria

Se encontró una mejoría de 18 puntos desde al diagnóstico a la post intervención, esta diferencia fue significativa y evidencia la efectividad del proceso de calidad.

Puntaje de auditoria comparado entre diagnostico e intervención

\begin{tabular}{|l|c|c|c|c|c|c|c|c|c|c|}
\hline & Obs & Medias & $\begin{array}{c}\text { Desviación } \\
\text { estándar }\end{array}$ & Min & $25 \%$ & Mediana & $75 \%$ & Max & Modo & $\begin{array}{c}\text { Prueba } \\
\mathrm{T}\end{array}$ \\
\hline Diagnostico & 346 & 69,5643 & 17,9816 & 15 & 58 & 70 & 82 & 100 & 70 & \\
\cline { 1 - 1 } $\begin{array}{l}\text { Post } \\
\text { intervención }\end{array}$ & 335 & 88,4224 & 15,2744 & 21,25 & 81 & 91,25 & 100 & 100 & 100 & 0,0000 \\
\hline
\end{tabular}

Discusión Los procesos de Auditoría Médica en Ecuador se han desarrollado en algunos subsistemas públicos y privados, el sector público ha tenido desarrollos heterogéneos en sus diferentes subsistemas, sin embargo, con la Red Publica en Salud y el Tarifario del Sistema Nacional de Salud existe una oportunidad de disminuir esa variabilidad.

Varios autores han coincidido que las intervenciones basadas en retroalimentación y que involucren actividades de docencia han dado resultados pequeños a moderados 13-19, incluso a nivel de terapia profesional a pacientes en el campo de la salud mental ha sido documentado, lo cual es coherente con la intervención que realizamos desde dos puntos de vista, el primero con el mejoramiento significativo en 6 meses de proceso y la nula conflictividad en el desarrollo del mismo. Paton ha demostrado que el simple hecho de tener datos e indicadores no ha generado mejoras sustanciales en calidad 20, Zhele propone que los cambios deben incidir en el comportamiento de los profesionales directamente 21 lo cual es coherente con la preocupación de varios investigadores sobre la no utilización de Guías Clínicas 22-24, en el estudio realizado, los indicadores son una parte del proceso, sin embargo, la mejora continua analizada antes y después es clave para la evolución de la gestión de la calidad.

La calidad de un proceso de auditoria medica se ve facilitado en un ambiente académico, se había analizado como un proceso de gestión clínica 25, sin embargo existe mucha evidencia de como la auditoria influye en ambientes académicos, directa e indirectamente 26-31, esto se da por varias razones: el ambiente académico en si, la visión del proceso de auditoria como una herramienta de aprendizaje, la evidencia de mejora con las intervenciones a manera de resolución de problemas; por lo tanto debería validarse la eficiencia del proceso para ser incluido en espacios de docencia de manera formal.

Las debilidades del proceso radican en la poca interoperabilidad de los sistemas informáticos, que incrementa el tiempo de revisión de los expedientes clínicos, además, que el proceso de auditoria médica no está incluido formalmente en los posgrados, sino que dependen de la gestión del tomador de decisiones de cada establecimiento de salud.

\section{Conclusiones}

La tendencia observada en todos los análisis (por fases o por meses), en general es de mejoramiento, incluso en aquellos ítems en los cuales se partía de una línea de base satisfactoria, esto se debe a la personalización de las intervenciones por la administración del hospital, los residentes y Auditoria, respondiendo a una intención de contextualización.

La aceptación del proceso por parte de los auditados es buena, la ausencia de conflictos es un indicador, aunque también se demuestra ausencia de apatía por la activa participación en procesos de discusiones de casos, esto se facilita por el ambiente de docencia y también a la natural adaptación del enfoque cooperativo y no punitivo de la estrategia de auditoria médica.

Este proceso de auditoría resultó más efectivo ya que además de cumplir el objetivo de mejoramiento de la calidad, incidió en un proceso académico y generó una cultura de calidad desde la formación temprana de médicos familiares.

Estos resultados son alentadores y confirman la validez, efectividad y eficiencia de los procesos de evaluación y monitoreo de la Calidad a través de Auditoria Medica integral en un medio docente. Es importante resaltar que el proceso debe ser continuo para lograr efectividad de la intervención. 


\section{Bibliografía}

1. Neuspiel DR, Stubbs EH, Liggin L. Improving Reporting of Outpatient Pediatric Medical Errors. Pediatrics. 15 de noviembre de 2011;peds.2011-0477.

2. Hysong SJ, Best RG, Pugh JA. Audit and feedback and clinical practice guideline adherence: Making feedback actionable. Implement Sci. 28 de abril de 2006;1:9.

3. Runyon KE. Some interactions between personality variables and management styles. J Appl Psychol. 1973;57(3):288-94.

4. Spector PE. Behavior in organizations as a function of employee's locus of control. Psychol Bull. 1982;91(3):482-97.

5. Mitchell TR, Smyser CM, Weed SE. Locus of Control: Supervision and Work Satisfaction. Acad Manage J. 1 de septiembre de 1975;18(3):623-31.

6. Katz FM, Snow R. Evaluación del rendimiento de los trabajadores de la salud: Manual de formación y supervisión. Cuad Salud Púbica OMS [Internet]. 1981 [citado 22 de noviembre de 2016];(72). Disponible en: http://bases.bireme.br/cgibin/wxislind.exe/iah/online/?

IsisScript $=$ iah/iah.xis\&src=google\&base=PAHO\&lang=p\&nextAction=lnk\&exprSearch=1619\&indexSearch=ID

7. Sedano AM. La supervisión educativa. Bordón Rev Pedagog. 1991;43(2):177-86.

8. Hysong DSJ. Meta-Analysis: Audit \& Feedback Features Impact Effectiveness on Care Quality. Med Care. marzo de 2009;47(3):356.

9. Neuspiel DR, Stubbs EH. Patient Safety in Ambulatory Care. Pediatr Clin North Am. diciembre de 2012;59(6):1341-54.

10. PhD SAS, MA JKO PhD, MEd, PhD KAR. The Handbook of Health Behavior Change, Third Edition. Springer Publishing Company; 2008. 860 p.

11. Fausto Gady Torres. Auditoria medica, La construcción de un proceso participativo de control de la calidad de la prestación en salud. En Chile; 2008.

12. Fausto Gady Torres. Informe Global de Auditoría médica de los Servicios de Salud en Quito. Quito: AUS/SODEM; 2008 p. 45. (AUS). Report No.: 1.

13. Jamtvedt G, Young JM, Kristoffersen DT, O’Brien MA, Oxman AD. Does telling people what they have been doing change what they do? A systematic review of the effects of audit and feedback. Qual Saf Health Care. diciembre de 2006;15(6):433-6.

14. O’Brien MA, Rogers S, Jamtvedt G, Oxman AD, Odgaard-Jensen J, Kristoffersen DT, et al. Educational outreach visits: effects on professional practice and health care outcomes. Cochrane Database Syst Rev. 17 de octubre de 2007;(4):CD000409.

15. Ivers N, Jamtvedt G, Flottorp S, Young JM, Odgaard-Jensen J, French SD, et al. Audit and feedback: effects on professional practice and healthcare outcomes. Cochrane Database Syst Rev. 13 de junio de 2012;(6):CD000259.

16. Forsetlund L, Bjørndal A, Rashidian A, Jamtvedt G, O’Brien MA, Wolf F, et al. Continuing education meetings and workshops: effects on professional practice and health care outcomes. Cochrane Database Syst Rev. 15 de abril de 2009;(2):CD003030.

17. Jamtvedt G, Young JM, Kristoffersen DT, O’Brien MA, Oxman AD. Audit and feedback: effects on professional practice and health care outcomes. Cochrane Database Syst Rev. 19 de abril de 2006;(2):CD000259.

18. Holloway EL, Neufeldt SA. Supervision: Its contributions to treatment efficacy. J Consult Clin Psychol. 1995;63(2):207-13.

19. Jr CEW. Does Psychotherapy Supervision Contribute to Patient Outcomes? Considering Thirty Years of Research. Clin Superv. 1 de julio de 2011;30(2):235-56.

20. Paton JY, Ranmal R, Dudley J, Committee on behalf of RCS. Clinical audit: Still an important tool for improving healthcare. Arch Dis Child - Educ Pract Ed. 1 de abril de 2015;100(2):83-8.

21. Zhelev Z, Abbott R, Rogers M, Fleming S, Patterson A, Hamilton WT, et al. Effectiveness of interventions to reduce ordering of thyroid function tests: a systematic review. BMJ Open. 1 de junio de 2016;6(6):e010065.

22. González-Juanatey JR, Alegría-Ezquerra E, Aznar-Costa J, Bertomeu-Martínez V, Franch-Nadal J, Palma-Gámiz JL. Conocimiento y aplicación de las guías de práctica clínica sobre riesgo cardiovascular en las consultas generales y especializadas. Rev Esp Cardiol. 1 de agosto de 2006;59(8):801-6.

23. S L, María L, Moore P. La medicina basada en evidencia: Visión después de una década. Rev Médica Chile. agosto de 2003;131(8):939-46.

24. Rello J, Lorente C, Bodí M, Diaz E, Ricart M, Kollef MH. Why do physicians not follow evidence-based guidelines for preventing ventilator-associated pneumonia?*: A survey based on the opinions of an international panel of intensivists. Chest. 1 de agosto de 2002;122(2):656-61.

25. Osorio S G, Sayes V N, Fernández M L, Araya C E, Poblete M D. Auditoria médica: herramienta de gestión moderna subvalorada. Rev Médica Chile. febrero de 2002;130(2):226-9. 
26. Gould BE, Grey MR, Huntington CG, Gruman C, Rosen JH, Storey E, et al. Improving patient care outcomes by teaching quality improvement to medical students in community-based practices. Acad Med J Assoc Am Med Coll. octubre de 2002;77(10):1011-8.

27. Holmboe ES, Prince L, Green M. Teaching and improving quality of care in a primary care internal medicine residency clinic. Acad Med J Assoc Am Med Coll. junio de 2005;80(6):571-7.

28. Rosenberg W, Donald A. Evidence based medicine: an approach to clinical problem-solving. BMJ. 29 de abril de 1995;310(6987):1122-6.

29. Thangaratinam S, Barnfield G, Weinbrenner S, Meyerrose B, Arvanitis TN, Horvath AR, et al. Teaching trainers to incorporate evidence-based medicine (EBM) teaching in clinical practice: the EU-EBM project. BMC Med Educ. 2009;9:59.

30. Rhodes DM, Ashcroft R, Atun RA, Freeman GK, Jamrozik K. Teaching evidence-based medicine to undergraduate medical students: a course integrating ethics, audit, management and clinical epidemiology. Med Teach. 1 de enero de 2006;28(4):313-7.

31. Linver MN, Paster SB, Rosenberg RD, Key CR, Stidley CA, King WV. Improvement in mammography interpretation skills in a community radiology practice after dedicated teaching courses: 2-year medical audit of 38,633 cases. Radiology. 1 de julio de 1992;184(1):39-43. 